\title{
Guy Thewes, Martin Uhrmacher (dir.), Extra Muros. Vorstädtische Räume in Spätmittelalter und früher Neuzeit
}

Pierre Monnet

\section{(2) OpenEdition \\ Journals}

Electronic version

URL: http://journals.openedition.org/ifha/10531

DOI: 10.4000/ifha.10531

ISSN: 2198-8943

Publisher

IFRA - Institut franco-allemand (sciences historiques et sociales)

Electronic reference

Pierre Monnet, « Guy Thewes, Martin Uhrmacher (dir.), Extra Muros. Vorstädtische Räume in Spätmittelalter und früher Neuzeit », Revue de l'IFHA [Online], Date of review, Online since 04 February 2020, connection on 24 September 2020. URL : http://journals.openedition.org/ifha/10531 ; DOI https://doi.org/10.4000/ifha.10531

This text was automatically generated on 24 September 2020.

(CIFHA 
Guy Thewes, Martin Uhrmacher (dir.), Extra Muros. Vorstädtische Räume in Spätmittelalter und früher Neuzeit

Pierre Monnet

\section{REFERENCES}

Guy Thewes, Martin Uhrmacher (dir.), Extra Muros. Vorstädtische Räume in Spätmittelalter und früher Neuzeit. Espaces suburbains au bas Moyen Âge et à l'époque moderne, Vienne/ Cologne/Weimar, Böhlau (Städteforschung, A/91), 521 p., 59,90€ 
Touchant l'espace urbain, notre imaginaire repose encore en grande partie sur l'une de ces dichotomies qui ont historiographiquement la peau dure, et autour desquelles se joue d'ailleurs le rapport complexe entre modernité et prémodernité : aujourd'hui, ce qui n'est pas faux, des mégalopoles, des métropoles, et même des cités de moindre importance aux contours flous, entourées d'espaces rurbanisés, aux transitions interminables; hier, des villes clairement délimitées, fortifiées, corsetées, séparées par leur droit et une claire conscience du dedans et du dehors de leurs campagnes ou plats pays.

C'est le principal mérite de ce volume issu d'un colloque tenu à l'université de

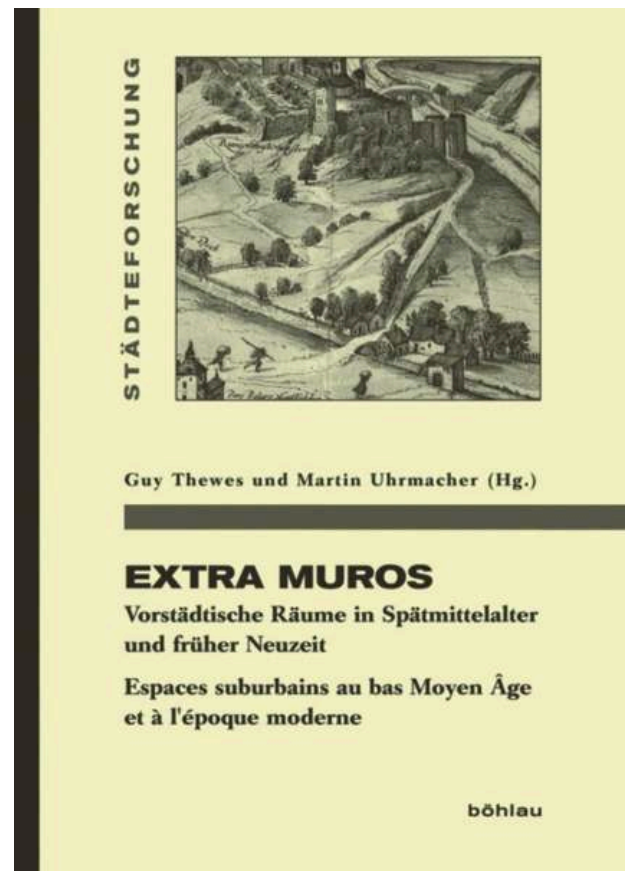
Luxembourg en 2013 que de reprendre à nouveaux frais la question, dans le droit fil des renouvellements méthodologiques portés par ce que l'on a pu appeler le «spatial turn». Que l'on regarde en effet la documentation normative ou pratique, juridique ou policière (car le faubourg est aussi le lieu de toutes les contrebandes), les comptes, les archives marchandes ou même les cartes anciennes, et aussitôt surgit au contraire l'image d'une ville prémoderne intrinsèquement et intensivement liée à ses faubourgs, ses productions agricoles environnantes ordonnées dans des vergers, des jardins, des vignes et des forêts administrés par la cité, ses routes, ses installations monumentales (du gibet au moulin avec une très belle contribution dédiée à l'emplacement des moulins dans les villes hongroises préindustrielles - en passant par les auberges à pèlerins ou les léproseries, les couvents suburbains, les tours fortifiées du périmètre défensif ou Landwehr...). Surtout, et c'est là l'apport fondamental du recueil, les réseaux sociaux urbains se sont également construits en fonction d'une circulation qui reliait l'intérieur et l'extérieur de la cité, à commencer par les micro-migrations journalières, la colonisation des villages par la rente et la commande artisanale puis industrielle, le système répandu des bourgeois forains, horsains et autres Ausbürger ou Pfahlbürger jouissant du droit urbain sans y résider. La diversité des cas, des espaces, des tailles de ville et des rythmes de croissance ou de décroissance a conduit les éditeurs scientifiques à ne pas s'enfermer dans un seul concept, laissant aux auteurs le soin de choisir tantôt espaces suburbains, tantôt espaces périurbains, tantôt banlieues, tant faubourgs, tantôt plat pays, tantôt périphérie, tantôt Umland, tantôt Vorstadt... pour désigner ces zones voisines de relation et de transition; et cela pour mieux cerner des cercles de plus ou moins grande densité des dépendances et des emprises urbaines sur les espaces environnants.

Le renouvellement majeur dont témoigne le volume tient à la prise en compte d'une sociologie de l'espace accouchant du récent concept d'espaces sociaux, une notion permettant de croiser les pratiques et les représentations spatiales au service d'une meilleure compréhension de la structuration des groupes sociaux. Pour ce faire, les 
19 contributions ont été ordonnées non pas en fonction de la géographie ou de la chronologie mais des variations thématiques et heuristiques introduites par le sujet. La représentation symbolique et cartographique d'abord, à l'image des villes des Pays-Bas croquées par Jacques de Deventer (milieu du XVI ${ }^{\mathrm{e}}$ siècle), de Lunebourg aux XV et XVI ${ }^{\mathrm{e}}$ siècles et de Bordeaux jusqu'au début du XIX ${ }^{e}$ siècle. On est frappé chaque fois par la précision et l'intensité de la représentation de faubourgs à ce point intégrés par la carte qu'ils deviennent consubstantiels de sa définition. Deux cas plus particuliers examinent sous le même angle l'influence du tracé et de la cartographie de la fortification sur la compréhension de la ville et de ses environs (Luxembourg et Mons).

Un second mouvement s'intéresse aux effets sociaux, disons plutôt sociotopographiques, et aux mécanismes de sociabilité induits par les relations entre la ville et ses faubourgs à l'exemple de Nuremberg, Munich, Bruxelles, Vienne, Lunebourg et de plusieurs villes du royaume de France, de Hongrie et de Pologne (Toruń, Chełmno, Poznań, Wrocław). Ces cas, analysés sous la triple perspective de l'inclusion, de l'exclusion et du contrôle, mettent au jour l'ambivalence et la complexité de ces espaces suburbains où se mêlent des étrangers, des exilés, des corps de métiers dévalorisés ou sales, des migrants et des voyageurs, des malades, mais où se trouvent aussi les espaces de villégiature, de repos, de festivité, de jeu et de délassement de la population urbaine mieux fixée et mieux intégrée, les jardins, palais d'été, villas contribuant à densifier cette zone périurbaine dans une sociabilité à la fois discriminante et partagée. C'est même là, plus encore qu'ailleurs, que se laissent observer de violents conflits pour la prise de possession des terrains, notamment à proximité des fleuves et des étangs.

Enfin, un troisième temps de l'étude est consacré aux modalités et aux degrés de l'influence urbaine, en privilégiant notamment l'analyse de la fiscalité et de sa répartition et la gestion des infrastructures (villes d'Italie, Augsbourg, Strasbourg) mais aussi, apport salutaire du volume, en descendant d'un échelon pour observer les mêmes phénomènes à l'œuvre dans des villes de petite taille, qui eurent aussi leurs faubourgs (Massif central, bourg monastique de Lagrasse).

Peut-être une conclusion aurait-elle pu relier tous les fils et ouvrir des perspectives, notamment en direction d'une étude sémantique du faubourg et de la diversité conceptuelle attachée à sa compréhension. Mais le recueil séduit par sa richesse, la variété des cas étendus à toute l'Europe, la présence de résumés en trois langues (allemand, français, anglais) et la profusion d'une cartographie précise et en couleurs.

\section{INDEX}

Chronological index: Moyen Âge

Subjects: Histoire des villes et des régions 